\title{
A SURVEY ON WEYL CALCULUS FOR REPRESENTATIONS OF NILPOTENT LIE GROUPS
}

\author{
INGRID BELTIŢă AND DANIEL BELTIŢĂ
}

\begin{abstract}
We survey some aspects of the pseudo-differential Weyl calculus for irreducible unitary representations of nilpotent Lie groups, ranging from the classical ideas to recently obtained results. The classical Weyl-Hörmander calculus is recovered for the Schrödinger representation of the Heisenberg group. Our discussion concerns various extensions of this classical situation to arbitrary nilpotent Lie groups and to some infinite-dimensional Lie groups that allow us to handle the magnetic pseudodifferential calculus.
\end{abstract}

\section{INTRODUCTION}

The Weyl calculus was first constructed in We28 for the purposes of quantum mechanics. It was afterwards investigated and extended to an abstract setting in An69] and An72. This calculus was also taken up in [Hor79] and made into a pseudo-differential calculus which plays a central role in the theory of partial differential equations and in many of its applications to mathematical physics.

In the present paper we provide a brief discussion of the Weyl calculus and its later extensions in the framework provided by nilpotent Lie groups and their representation theory, ranging from some classical ideas of [We28] and [Hor79], going through the important developments in [Pe94], and concluding by recent results from $\mathrm{BB} 09 \mathrm{a}, \mathrm{BB} 09 \mathrm{~b}$, and $[\mathrm{BB} 09 \mathrm{c}$. We shall emphasize the role of the coadjoint orbits and the corresponding unitary representations as a natural background for the Weyl calculus. For instance the classical phase space $\mathbb{R}^{n} \times \mathbb{R}^{n}$ should be thought of as a coadjoint orbit of the Heisenberg group, corresponding to the Schrödinger representation, which leads to a transparent description of the classical Weyl calculus on $\mathbb{R}^{n}$ as a very special case of the calculus constructed in Pe94 for arbitrary nilpotent Lie groups (see Sections 2 and 3 below). The same idea allowed us to show in BB09a that the magnetic pseudo-differential calculus of MP04 is governed by a certain infinite-dimensional Lie group, in the sense that it can be constructed as a Weyl quantization of a certain finite-dimensional coadjoint orbit of that group and the symbol spaces for the magnetic calculus are actually function spaces on that orbit (see Section 4).

For the sake of simplicity let us mention here that there exist other interesting lines of investigation on symbol calculi for nilpotent Lie groups, which have however a different flavour and therefore we do not discuss them here; see for instance the papers [Me83, [How84] and Gl07. See also [Mi82, [Mi86], HRW84, and Ma91 for examples and remarks on the relationship between a symbol calculus and a coadjoint orbit.

Notation. Throughout the paper we denote by $\mathscr{S}(\mathscr{V})$ the Schwartz space on a finite-dimensional real vector space $\mathscr{V}$. That is, $\mathscr{S}(\mathscr{V})$ is the set of all smooth functions that decay faster than any polynomial together with their partial derivatives of arbitrary order. Its topological dual - the space of tempered distributions on $\mathscr{V}$ - is denoted by $\mathscr{S}^{\prime}(\mathscr{V})$. We use the notation $\mathscr{C}_{\text {pol }}^{\infty}(\mathscr{V})$ for the space of smooth functions

2000 Mathematics Subject Classification. Primary 81S30; Secondary 22E25,22E27,35S05.

Key words and phrases. Weyl calculus; magnetic field; nilpotent Lie group; semidirect product. 
that grow polynomially together with their partial derivatives of arbitrary order. In Section 2 we shall also have the occasion to use these notions with $\mathscr{V}$ replaced by a coadjoint orbit of a nilpotent Lie group. In this situation we need the notion of polynomial structure on a manifold; see Sect. 1 in [Pe89] for details. We use $\langle\cdot, \cdot\rangle$ to denote any duality pairing between finite-dimensional real vector spaces whose meaning is clear from the context. The Lebesgue measures on linear spaces (or Liouville measures on coadjoint orbits) and on their duals will always be normalized such that the corresponding Fourier transforms are unitary operators on the $L^{2}$-spaces.

\section{The Classical pseudo-Differential Weyl Calculus on $\mathbb{R}^{n}$}

2.1. The earlier perspective on the Weyl calculus. The Weyl calculus was introduced in We28 as a quantization procedure, that is, a natural correspondence between the classical observables and the quantum ones. More specifically, let $P_{1}, \ldots, P_{n}$ be the quantum momentum operators and $Q_{1}, \ldots, Q_{n}$ the quantum position operators in $L^{2}\left(\mathbb{R}^{n}\right)$. Recall that for $f \in \mathscr{S}\left(\mathbb{R}^{n}\right)$ and a generic point $q=\left(q_{1}, \ldots, q_{n}\right) \in$ $\mathbb{R}^{n}$ we have for $j=1, \ldots, n$,

$$
\left(Q_{j} f\right)(q)=q_{j} f(q) \text { and } P_{j} f=\frac{1}{\mathrm{i}} \frac{\partial f}{\partial q_{j}} .
$$

One of the remarkable properties of these operators is that for arbitrary points $p=\left(p_{1}, \ldots, p_{n}\right) \in \mathbb{R}^{n}$ and $q=\left(q_{1}, \ldots, q_{n}\right) \in \mathbb{R}^{n}$ the linear combination

$$
p \cdot Q+q \cdot P:=p_{1} Q_{1}+\cdots+p_{n} Q_{n}+q_{1} P_{1}+\cdots+q_{n} P_{n}
$$

defines a self-adjoint operator in $L^{2}\left(\mathbb{R}^{n}\right)$, which in turn gives rise to a unitary operator $\exp (\mathrm{i}(p \cdot Q+q \cdot P))$ that leaves $\mathscr{S}\left(\mathbb{R}^{n}\right)$ invariant.

Now we can use these remarks to make the following definition of the pseudo-differential WeylHörmander calculus on $\mathbb{R}^{n}$ (see [Hor79]).

Definition 2.1. For every $a \in \mathscr{S}\left(\mathbb{R}^{n} \times \mathbb{R}^{n}\right)$ we define the corresponding pseudo-differential operator $a(Q, P)$ by

$$
a(Q, P) f=\iint_{\mathbb{R}^{n} \times \mathbb{R}^{n}} \widehat{a}(p, q) \exp (\mathrm{i}(p \cdot Q+q \cdot P)) f \mathrm{~d} p \mathrm{~d} q
$$

for arbitrary $f \in \mathscr{S}\left(\mathbb{R}^{n}\right)$, where $\widehat{a} \in \mathscr{S}\left(\mathbb{R}^{n} \times \mathbb{R}^{n}\right)$ stands for the Fourier transform of the symbol a.

Remark 2.2. For every tempered distribution $a \in \mathscr{S}^{\prime}\left(\mathbb{R}^{n} \times \mathbb{R}^{n}\right)$ we can interpret the integral (2.1) in the distributional sense and thus define the corresponding pseudo-differential operator $a(Q, P): \mathscr{S}\left(\mathbb{R}^{n}\right) \rightarrow$ $\mathscr{S}^{\prime}\left(\mathbb{R}^{n}\right)$. One can also compute the distribution kernel of the latter operator. More specifically, we have

$$
(a(Q, P) f)(q)=\iint_{\mathbb{R}^{n} \times \mathbb{R}^{n}} a\left(\frac{q+q^{\prime}}{2}, p\right) e^{i\left(q-q^{\prime}\right) \cdot p} f\left(q^{\prime}\right) \mathrm{d} p \mathrm{~d} q^{\prime}
$$

for $f \in \mathscr{S}\left(\mathbb{R}^{n}\right)$ (see [Hor07] for more details).

2.2. Weyl calculus from the perspective of the Heisenberg group. A particularly deep insight into the Weyl calculus of Definition 2.1 comes from taking into account the commutation relations satisfied by the operators $Q_{1}, \ldots, Q_{n}$ and $P_{1}, \ldots, P_{n}$, namely

$$
\left[Q_{j}, Q_{k}\right]=\left[P_{j}, P_{k}\right]=0 \text { and }\left[Q_{j}, P_{k}\right]=\delta_{j k} \mathrm{i} \cdot I
$$

where $\delta_{j k}$ stands for the Kronecker's delta and $I$ denotes the identity operator on $L^{2}\left(\mathbb{R}^{n}\right)$. It follows from these commutation relations that we actually have to deal with the Schrödinger representation of the Heisenberg group from the following definition. 
Definition 2.3. For every integer $n \geq 1$ let '.' denote the Euclidean scalar product on $\mathbb{R}^{n}$. We introduce the Heisenberg algebra $\mathfrak{h}_{2 n+1}=\mathbb{R}^{n} \times \mathbb{R}^{n} \times \mathbb{R}$ with the bracket

$$
\left[(q, p, t),\left(q^{\prime}, p^{\prime}, t^{\prime}\right)\right]=\left[\left(0,0, p \cdot q^{\prime}-p^{\prime} \cdot q\right)\right] .
$$

The Heisenberg group $\mathbb{H}_{2 n+1}$ is just $\mathfrak{h}_{2 n+1}$ thought of as a group with the multiplication $*$ defined by

$$
X * Y=X+Y+\frac{1}{2}[X, Y] .
$$

The unit element is $0 \in \mathbb{H}_{2 n+1}$ and the inversion mapping given by $X^{-1}:=-X$.

See also How80, Fo89, FG92 and Ch. 9 in Gr01 for discussions on the Heisenberg group and its importance for the harmonic analysis on $\mathbb{R}^{n}$.

To define the Schrödinger representation we first exhibit the Heisenberg group as a semidirect product and then use a natural representation of that semidirect product. The details are recorded in the following remark.

Remark 2.4. Consider $\mathbb{R}^{n+1} \simeq \mathbb{R}^{n} \times \mathbb{R}$ and the natural representation

$$
\rho:\left(\mathbb{R}^{n},+\right) \rightarrow \text { End }\left(\mathbb{R}^{n+1}\right), \quad \rho(q)(p, t)=(p, p \cdot q+t) .
$$

Then it is straightforward to check that the mapping

$$
\Psi: \mathbb{H}_{2 n+1} \rightarrow \mathbb{R}^{n+1} \rtimes_{\rho} \mathbb{R}^{n}, \quad(q, p, t) \mapsto\left(\left(p, \frac{1}{2} p \cdot q+t\right), q\right)
$$

is an isomorphism of Lie groups.

Let $\mathscr{P}_{1}\left(\mathbb{R}^{n}\right)$ be the linear space of real polynomial functions of degree $\leq 1$ on $\mathbb{R}^{n}$ and note that $\mathscr{P}_{1}\left(\mathbb{R}^{n}\right)$ is linearly isomorphic to $\mathbb{R}^{n} \times \mathbb{R}$, since for every $\varphi \in \mathscr{P}_{1}\left(\mathbb{R}^{n}\right)$ there exist uniquely determined $\xi \in \mathbb{R}^{n}$ and $t \in \mathbb{R}$ such that $\varphi(x)=\xi \cdot x+t$ for every $x \in \mathbb{R}^{n}$. We also get a linear representation

$$
\rho:\left(\mathbb{R}^{n},+\right) \rightarrow \operatorname{End}\left(\mathscr{P}_{1}\left(\mathbb{R}^{n}\right)\right), \quad \rho(q) \varphi=\varphi(q+\cdot)
$$

and then $\mathbb{H}_{2 n+1} \simeq \mathscr{P}_{1}\left(\mathbb{R}^{n}\right) \rtimes_{\rho} \mathbb{R}^{n}$ in view of the above paragraph (see also Ex. 2.6 in [BB09a]).

Note that the semidirect product $\mathscr{P}_{1}\left(\mathbb{R}^{n}\right) \rtimes_{\rho} \mathbb{R}^{n}$ has a natural unitary representation on $L^{2}\left(\mathbb{R}^{n}\right)$ given for arbitrary $(\varphi, q) \in \mathscr{P}_{1}\left(\mathbb{R}^{n}\right) \rtimes_{\rho} \mathbb{R}^{n}$ by

$$
\left(\forall f \in L^{2}\left(\mathbb{R}^{n}\right)\right) \quad \pi(\varphi, q) f=\mathrm{e}^{\mathrm{i} \varphi(\cdot)} f(q+\cdot) .
$$

Then the above explicit isomorphisms $\mathbb{H}_{2 n+1} \simeq \mathbb{R}^{n+1} \rtimes_{\rho} \mathbb{R}^{n} \simeq \mathscr{P}_{1}\left(\mathbb{R}^{n}\right) \rtimes_{\rho} \mathbb{R}^{n}$, lead to the unitary representation $\pi: \mathbb{H}_{2 n+1} \rightarrow \mathscr{B}\left(L^{2}\left(\mathbb{R}^{n}\right)\right)$ defined by

$$
(\pi(q, p, t) f)(x)=\mathrm{e}^{\mathrm{i}\left(p \cdot x+\frac{1}{2} p \cdot q+t\right)} f(q+x) \text { for a.e. } x \in \mathbb{R}^{n}
$$

for arbitrary $f \in L^{2}\left(\mathbb{R}^{n}\right)$ and $(q, p, t) \in \mathbb{H}_{2 n+1}$. This is the Schrödinger representation of the Heisenberg group $\mathbb{H}_{2 n+1}$.

The Schrödinger representation of the Heisenberg group provides the natural background for the pseudo-differential Weyl calculus on $\mathbb{R}^{n}$. To illustrate this idea, we shall describe a condition on the symbol $a \in \mathscr{S}^{\prime}\left(\mathbb{R}^{n} \times \mathbb{R}^{n}\right)$ which ensures that the pseudo-differential operator $\operatorname{Op}(a)$ is bounded on $L^{2}\left(\mathbb{R}^{n}\right)$. This $L^{2}$-boundedness theorem was obtained in [GH99] and is stated in terms of the modulation spaces introduced below.

Definition 2.5. Let us denote by $\langle\cdot, \cdot\rangle: \mathscr{S}^{\prime}\left(\mathbb{R}^{n}\right) \times \mathscr{S}\left(\mathbb{R}^{n}\right) \rightarrow \mathbb{C}$ the usual duality pairing. Assume that $1 \leq r, s \leq \infty$ and $\phi \in \mathscr{S}\left(\mathbb{R}^{n}\right)$ and define for every tempered distribution $b \in \mathscr{S}^{\prime}\left(\mathbb{R}^{n}\right)$ the corresponding ambiguity function

$$
\mathscr{A}_{\phi} b: \mathbb{R}^{n} \times \mathbb{R}^{n} \rightarrow \mathbb{C}, \quad\left(\mathscr{A}_{\phi} b\right)(q, p)=\langle b, \overline{\pi(q, p, 0) \phi}\rangle
$$


and then

$$
\|b\|_{M_{\phi}^{r, s}}=\left(\int_{\mathbb{R}^{n}}\left(\int_{\mathbb{R}^{n}}\left|\left(\mathscr{A}_{\phi} b\right)(q, p)\right|^{s} \mathrm{~d} q\right)^{r / s} \mathrm{~d} p\right)^{1 / r} \in[0, \infty]
$$

with the usual conventions if $r$ or $s$ is infinite. Then the space

$$
M^{r, s}\left(\mathbb{R}^{n}\right):=\left\{b \in \mathscr{S}^{\prime}\left(\mathbb{R}^{n}\right) \mid\|b\|_{M_{\phi}^{r, s}}<\infty\right\}
$$

does not depend on $\phi \in \mathscr{S}\left(\mathbb{R}^{n}\right)$ and is called a modulation space on $\mathbb{R}^{n}$.

Theorem 2.6. For every $a \in M^{\infty, 1}\left(\mathbb{R}^{2 n}\right)$ the corresponding pseudo-differential operator $\operatorname{Op}(a)$ is bounded on $L^{2}\left(\mathbb{R}^{n}\right)$. Moreover, for every $\phi \in \mathscr{S}\left(\mathbb{R}^{2 n}\right)$ there exists a constant $C_{\phi}>0$ such that if $a \in M^{\infty, 1}\left(\mathbb{R}^{2 n}\right)$, then $\|\mathrm{Op}(a)\| \leq C_{\phi}\|a\|_{M_{\phi}^{1, \infty}}$.

Proof. See Th. 1.1 in GH99.

\section{WEyl CALCUlus FOR IRREDUCiBle UNitARY REPRESENTATIONS}

We shall briefly describe some of the remarkable results obtained in [Pe94] (relying on [Pe84, Pe88, and $[$ Pe89])) on the Weyl calculus for irreducible unitary representations of nilpotent Lie groups.

\subsection{Preduals for coadjoint orbits.}

Setting 3.1. We shall use the following notation:

(1) Let $G$ be a connected, simply connected, nilpotent Lie group with the Lie algebra $\mathfrak{g}$. Then the exponential map $\exp _{G}: \mathfrak{g} \rightarrow G$ is a diffeomorphism with the inverse denoted by $\log _{G}: G \rightarrow \mathfrak{g}$.

(2) We denote by $\mathfrak{g}^{*}$ the linear dual space to $\mathfrak{g}$ and by $\langle\cdot, \cdot\rangle: \mathfrak{g}^{*} \times \mathfrak{g} \rightarrow \mathbb{R}$ the natural duality pairing.

(3) Let $\xi_{0} \in \mathfrak{g}^{*}$ with the corresponding coadjoint orbit $\mathscr{O}:=\operatorname{Ad}_{G}^{*}(G) \xi_{0} \subseteq \mathfrak{g}^{*}$.

(4) The isotropy group at $\xi_{0}$ is $G_{\xi_{0}}:=\left\{g \in G \mid \operatorname{Ad}_{G}^{*}(g) \xi_{0}=\xi_{0}\right\}$ with the corresponding isotropy Lie algebra $\mathfrak{g}_{\xi_{0}}=\left\{X \in \mathfrak{g} \mid \xi_{0} \circ \operatorname{ad}_{\mathfrak{g}} X=0\right\}$. If we denote the center of $\mathfrak{g}$ by $\mathfrak{z}:=\{X \in \mathfrak{g} \mid[X, \mathfrak{g}]=\{0\}\}$, then it is clear that $\mathfrak{z} \subseteq \mathfrak{g}_{\xi_{0}}$.

(5) Let $n:=\operatorname{dim} \mathfrak{g}$ and fix a sequence of ideals in $\mathfrak{g}$,

$$
\{0\}=\mathfrak{g}_{0} \subset \mathfrak{g}_{1} \subset \cdots \subset \mathfrak{g}_{n}=\mathfrak{g}
$$

such that $\operatorname{dim}\left(\mathfrak{g}_{j} / \mathfrak{g}_{j-1}\right)=1$ and $\left[\mathfrak{g}, \mathfrak{g}_{j}\right] \subseteq \mathfrak{g}_{j-1}$ for $j=1, \ldots, n$.

(6) Pick any $X_{j} \in \mathfrak{g}_{j} \backslash \mathfrak{g}_{j-1}$ for $j=1, \ldots, n$, so that the set $\left\{X_{1}, \ldots, X_{n}\right\}$ will be a Jordan-Hölder basis in $\mathfrak{g}$.

Definition 3.2. Consider the set of jump indices of the coadjoint orbit $\mathscr{O}$ with respect to the aforementioned Jordan-Hölder basis $\left\{X_{1}, \ldots, X_{n}\right\} \subset \mathfrak{g}$,

$$
e:=\left\{j \in\{1, \ldots, n\} \mid \mathfrak{g}_{j} \not \subset \mathfrak{g}_{j-1}+\mathfrak{g}_{\xi_{0}}\right\}=\left\{j \in\{1, \ldots, n\} \mid X_{j} \notin \mathfrak{g}_{j-1}+\mathfrak{g}_{\xi_{0}}\right\}
$$

and then define the corresponding predual of the coadjoint orbit $\mathscr{O}$,

$$
\mathfrak{g}_{e}:=\operatorname{span}\left\{X_{j} \mid j \in J_{\xi_{0}}\right\} \subseteq \mathfrak{g} .
$$

We note the direct sum decomposition $\mathfrak{g}=\mathfrak{g}_{\xi_{0}} \dot{+} \mathfrak{g}_{e}$. 
Remark 3.3. Let $\left\{\xi_{1}, \ldots, \xi_{n}\right\} \subset \mathfrak{g}^{*}$ be the dual basis for $\left\{X_{1}, \ldots, X_{n}\right\} \subset \mathfrak{g}$. Then the coadjoint orbit $\mathscr{O}$ can be described in terms of the jump indices mentioned in Definition 3.2. More specifically, if we denote

$$
\mathfrak{g}_{\mathscr{O}}^{*}:=\operatorname{span}\left\{\xi_{j} \mid j \in e\right\} \quad \text { and } \quad \mathfrak{g}_{\mathscr{O}}^{\perp}:=\operatorname{span}\left\{\xi_{j} \mid j \notin e\right\},
$$

then the coadjoint orbit $\mathscr{O} \subset \mathfrak{g}^{*} \simeq \mathfrak{g}_{e}^{*} \times \mathfrak{g}_{e}^{\perp}$ is the graph of a certain polynomial mapping $\mathfrak{g}_{e}^{*} \rightarrow \mathfrak{g}_{e}^{\perp}$. This leads to the following pieces of information on $\mathscr{O}$ :

(1) $\operatorname{dim} \mathscr{O}=\operatorname{dim} \mathfrak{g}_{e}=\operatorname{card} e=: d$;

(2) if we let $j_{1}<\cdots<j_{d}$ such that $e=\left\{j_{1}, \ldots, j_{d}\right\}$, then the mapping

$$
\mathscr{O} \rightarrow \mathbb{R}^{d}, \quad \xi \rightarrow\left(\left\langle\xi, X_{j_{1}}\right\rangle, \ldots,\left\langle\xi, X_{j_{d}}\right\rangle\right)
$$

is a global chart which takes the Liouville measure of $\mathscr{O}$ to a Lebesgue measure on $\mathbb{R}^{d}$.

See for instance Lemma 1.6.1 in [Pe89] for more details and proofs for these assertions.

\subsection{Weyl calculus for unitary irreducible representations.}

Setting 3.4. In addition to Setting 3.1 we now fix some further notation:

(1) Let $\pi: G \rightarrow \mathscr{B}(\mathscr{H})$ be any unitary irreducible representations associated with the coadjoint orbit $\mathscr{O}$ by Kirillov's theorem ( Kir62 $)$.

(2) We define the Fourier transform $\mathscr{S}(\mathscr{O}) \rightarrow \mathscr{S}\left(\mathfrak{g}_{e}\right)$ by

$$
\left(\forall X \in \mathfrak{g}_{e}\right) \quad \widehat{a}(X)=\int_{\mathscr{O}} \mathrm{e}^{-\mathrm{i}\langle\xi, X\rangle} a(\xi) \mathrm{d} \xi
$$

for every $a \in \mathscr{S}(\mathscr{O})$, where $\mathrm{d} \xi$ stands for a Liouville measure on $\mathscr{O}$ (see also Remark 3.3). This Fourier transform is invertible (Lemma 4.1.1 in [Pe94]) and its inverse is denoted by $\mathscr{S}\left(\mathfrak{g}_{e}\right) \rightarrow$ $\mathscr{S}(\mathscr{O}), a \mapsto \check{a}$.

Remark 3.5. Let us consider the space of smooth vectors for the representation $\pi$,

$$
\mathscr{H}_{\infty}:=\left\{v \in \mathscr{H} \mid \pi(\cdot) v \in \mathscr{C}^{\infty}(G, \mathscr{H})\right\} .
$$

Then $\mathscr{H}_{\infty}$ has a natural structure of Fréchet space which carries the derivate representation $\mathrm{d} \pi: \mathfrak{g} \rightarrow$ End $\left(\mathscr{H}_{\infty}\right)$. The latter map is a homomorphism of Lie algebras defined by

$$
\left(\forall X \in \mathfrak{g}, v \in \mathscr{H}_{\infty}\right) \quad \mathrm{d} \pi(X) v=\left.\frac{\mathrm{d}}{\mathrm{d} t}\right|_{t=0} \pi\left(\exp _{G}(t X)\right) v .
$$

Now let us denote by $\mathfrak{S}_{p}(\mathscr{H})$ the Schatten ideals of operators on $\mathscr{H}$ for $1 \leq p \leq \infty$. Consider the unitary representation $\Pi: G \times G \rightarrow \mathscr{B}\left(\mathfrak{S}_{2}(\mathscr{H})\right)$ defined by

$$
\left(\forall g_{1}, g_{2} \in G\right)\left(\forall T \in \mathfrak{S}_{2}(\mathscr{H})\right) \quad \Pi\left(g_{1}, g_{2}\right) T=\pi\left(g_{1}\right) T \pi\left(g_{2}\right)^{-1} .
$$

It is not difficult to see that $\Pi$ is strongly continuous. The corresponding space of smooth vectors is denoted by $\mathscr{B}(\mathscr{H})_{\infty}$ and is called the space of smooth operators for the representation $\pi$. One can prove that actually $\mathscr{B}(\mathscr{H})_{\infty} \subseteq \mathfrak{S}_{1}(\mathscr{H})$.

For an alternative description of $\mathscr{B}(\mathscr{H})_{\infty}$ let $\mathfrak{g}_{\mathbb{C}}:=\mathfrak{g} \otimes_{\mathbb{R}} \mathbb{C}$ be the complexification of $\mathfrak{g}$ with the corresponding universal associative enveloping algebra $\mathrm{U}\left(\mathfrak{g}_{\mathbb{C}}\right)$. Then the aforementioned homomorphism of Lie algebras $\mathrm{d} \pi$ has a unique extension to a homomorphism of unital associative algebras $\mathrm{d} \pi: \mathrm{U}\left(\mathfrak{g}_{\mathbb{C}}\right) \rightarrow$ End $\left(\mathscr{H}_{\infty}\right)$. One can prove that for $T \in \mathscr{B}(\mathscr{H})$ we have $T \in \mathscr{B}(\mathscr{H})_{\infty}$ if and only if $T(\mathscr{H})+T^{*}(\mathscr{H}) \subseteq \mathscr{H}_{\infty}$ and $\mathrm{d} \pi(u) T, \mathrm{~d} \pi(u) T^{*} \in \mathscr{B}(\mathscr{H})$ for every $u \in \mathrm{U}\left(\mathfrak{g}_{\mathbb{C}}\right)$. (See subsect. 1.2 in [Pe94].) 
Definition 3.6. The Weyl calculus $\mathrm{Op}^{\pi}(\cdot)$ for the unitary representation $\pi$ is defined for every $a \in \mathscr{S}(\mathscr{O})$ by

$$
\operatorname{Op}^{\pi}(a)=\int_{\mathfrak{g}_{e}} \widehat{a}(X) \pi\left(\exp _{G} X\right) \mathrm{d} X \in \mathscr{B}(\mathscr{H}) .
$$

We call $\mathrm{Op}^{\pi}(a)$ is the pseudo-differential operator with the symbol $a \in \mathscr{S}(\mathscr{O})$.

Theorem 3.7. The Weyl calculus has the following properties:

(1) For every symbol $a \in \mathscr{S}(\mathscr{O})$ we have $\mathrm{Op}^{\pi}(a) \in \mathscr{B}(\mathscr{H})_{\infty}$ and the mapping $\mathscr{S}(\mathscr{O}) \rightarrow \mathscr{B}(\mathscr{H})_{\infty}$, $a \mapsto \mathrm{Op}^{\pi}(a)$ is a linear topological isomorphism.

(2) For every $T \in \mathscr{B}(\mathscr{H})_{\infty}$ we have $T=\mathrm{Op}^{\pi}(a)$, where a $\in \mathscr{S}(\mathscr{O})$ satisfies the condition $\widehat{a}(X)=$ $\operatorname{Tr}\left(\pi\left(\exp _{G} X\right)^{-1} A\right)$ for every $X \in \mathfrak{g}_{e}$.

(3) For every $a, b \in \mathscr{S}(\mathscr{O})$ we have

(a) $\mathrm{Op}^{\pi}(\bar{a})=\mathrm{Op}^{\pi}(a)^{*}$;

(b) $\operatorname{Tr}\left(\mathrm{Op}^{\pi}(a)\right)=\int_{\mathscr{O}} a(\xi) \mathrm{d} \xi$;

(c) $\operatorname{Tr}\left(\mathrm{Op}^{\pi}(a) \mathrm{Op}^{\pi}(b)\right)=\int_{\mathscr{O}} a(\xi) b(\xi) \mathrm{d} \xi ;$

(d) $\operatorname{Tr}\left(\mathrm{Op}^{\pi}(a) \mathrm{Op}^{\pi}(b)^{*}\right)=\int_{\mathscr{O}} a(\xi) \overline{b(\xi)} \mathrm{d} \xi$.

Proof. See Th. 4.1.4 and Th. 2.2.7 in Pe94.

Remark 3.8. Let $\mathscr{B}(\mathscr{H})_{\infty}^{*}$ be the topological dual of the Fréchet space $\mathscr{B}(\mathscr{H})_{\infty}$ and denote by $\langle\cdot, \cdot\rangle$ either of the duality pairings

$$
\mathscr{B}(\mathscr{H})_{\infty}^{*} \times \mathscr{B}(\mathscr{H})_{\infty} \rightarrow \mathbb{C} \text { and } \mathscr{S}^{\prime}(\mathscr{O}) \times \mathscr{S}(\mathscr{O}) \rightarrow \mathbb{C} .
$$

Then for every tempered distribution $a \in \mathscr{S}^{\prime}(\mathscr{O})$ we can use Theorem 3.7(1) to define $\operatorname{Op}^{\pi}(a) \in \mathscr{B}(\mathscr{H})_{\infty}^{*}$ such that

$$
(\forall b \in \mathscr{S}(\mathscr{O})) \quad\left\langle\mathrm{Op}^{\pi}(a), \mathrm{Op}^{\pi}(b)\right\rangle=\langle a, b\rangle
$$

Just as in Definition 3.6 we call $\mathrm{Op}^{\pi}(a)$ the pseudo-differential operator with the symbol $a \in \mathscr{S}^{\prime}(\mathscr{O})$. Note that if actually $a \in \mathscr{S}(\mathscr{O})$, then the present notation agrees with Definition 3.6 because of Theorem 3.7(3c).

The continuity properties of the above pseudo-differential operators can be investigated by using modulation spaces of symbols; see [BB09c for details. Specifically, one can extend Definition 2.5 in order to introduce modulation spaces $M_{\phi}^{r, s}(\pi)$ for every unitary irreducible representation $\pi: G \rightarrow \mathscr{B}(\mathscr{H})$. We always have $\mathscr{H}_{\infty} \subseteq M_{\phi}^{r, s}(\pi)$ and $M_{\phi}^{2,2}(\pi)=\mathscr{H}$. If the representation $\pi$ is square-integrable modulo the center of $G$ and $\mathscr{O}$ is the corresponding coadjoint orbit, then there exists a natural representation $\pi^{\#}: G \ltimes G \rightarrow \mathscr{B}\left(L^{2}(\mathscr{O})\right)$ such that the Weyl calculus $\mathrm{Op}^{\pi}(\cdot)$ defines a continuous linear mapping from the modulation space $M^{\infty, 1}\left(\pi^{\#}\right)$ into the space of bounded linear operators on $\mathscr{H}$. Theorem 2.6 is recovered in the special case when $\pi$ is the Schrödinger representation of the Heisenberg group $\mathbb{H}_{2 n+1}$.

In the following statement we shall use the notation

$$
(\forall X \in \mathfrak{g}) \quad \psi^{X}: \mathscr{O} \rightarrow \mathbb{C}, \quad \psi^{X}(\xi)=\mathrm{i}\langle\xi, X\rangle .
$$

For every integer $m \geq 0$ and every $X \in \mathfrak{g}$ the $m$-th power $\left(\psi^{X}\right)^{m}$ can be thought of as an element in $\mathscr{S}^{\prime}(\mathscr{O})$ in the usual way.

Theorem 3.9. The Weyl calculus with symbols in $\mathscr{S}^{\prime}(\mathscr{O})$ has the following properties:

(1) The mapping $\mathscr{S}^{\prime}(\mathscr{O}) \rightarrow \mathscr{B}(\mathscr{H})_{\infty}^{*}, a \mapsto \mathrm{Op}^{\pi}(a)$ is a linear topological isomorphism. 
(2) For every $X \in \mathfrak{g}$ we have $\mathrm{Op}^{\pi}\left(\psi^{X}\right)=\mathrm{d} \pi(X)$.

(3) If $Y \in \mathfrak{g}_{e}$, then for every integer $m \geq 0$ we have $\mathrm{Op}^{\pi}\left(\left(\psi^{Y}\right)^{m}\right)=\mathrm{d} \pi(Y)^{m}$.

Proof. See Th. 4.1.4(7)-(8) in [Pe94.

Definition 3.10. Note that $\mathscr{B}(\mathscr{H})_{\infty}$ is an involutive associative subalgebra of $\mathscr{B}(\mathscr{H})$ as an easy consequence of the alternative description in Remark 3.5. It then follows by Theorem 3.711) that there exists an uniquely defined bilinear associative Moyal product

$$
\mathscr{S}(\mathscr{O}) \times \mathscr{S}(\mathscr{O}) \rightarrow \mathscr{S}(\mathscr{O}), \quad(a, b) \mapsto a \#^{\pi} b
$$

such that

$$
(\forall a, b \in \mathscr{S}(\mathscr{O})) \quad \mathrm{Op}^{\pi}\left(a \#^{\pi} b\right)=\mathrm{Op}^{\pi}(a) \mathrm{Op}^{\pi}(b) .
$$

Thus $\mathscr{S}(\mathscr{O})$ is made into an involutive associative algebra such that the mapping $\mathscr{S}(\mathscr{O}) \rightarrow \mathscr{B}(\mathscr{H})_{\infty}$, $a \mapsto \mathrm{Op}^{\pi}(a)$ is an algebra isomorphism.

Remark 3.11. It was proved in Ma07 that if $\pi$ is a square-integrable representation, then the Moyal product $\#^{\pi}$ is a star product and an explicit formal expansion was obtained.

Remark 3.12. In the case of the Heisenberg group (see Definition 2.3), let us perform the identification $\mathfrak{h}_{2 n+1}^{*} \simeq \mathbb{R}^{n} \times \mathbb{R}^{n} \times \mathbb{R}$ by means of the Euclidean structure of $\mathbb{R}^{2 n+1}$. Then the only nontrivial coadjoint orbits are of the form $\mathscr{O}=\mathbb{R}^{n} \times \mathbb{R}^{n} \times\{t\}$ with $t \in \mathbb{R} \backslash\{0\}$, and $\mathscr{S}(\mathscr{O})$ can be naturally identified with $\mathscr{S}\left(\mathbb{R}^{n} \times \mathbb{R}^{n}\right)$. When $t=1$, the corresponding irreducible representation of $\mathbb{H}_{2 n+1}$ is given by (2.3), while the predual to the coadjoint orbit can be identified to $\mathbb{R}^{n} \times \mathbb{R}^{n}$. Then it is easy to see that

$$
a(Q, P)=\mathrm{Op}^{\pi}(a) \quad \text { for } \quad a \in \mathscr{S}\left(\mathbb{R}^{n} \times \mathbb{R}^{n}\right) .
$$

Thus the classical Weyl pseudo-differential calculus on $\mathbb{R}^{n}$ can be directly obtained as a special calculus of the Weyl calculus of [Pe94] described in Definition 3.6] above.

\section{Magnetic Weyl calculus on nilpotent Lie groups}

The magnetic pseudo-differential Weyl calculus on $\mathbb{R}^{n}$ developed in MP04, IMP07 and other works was motivated by problems in quantum mechanics. In the present section we describe results from BB09a (see also [BB09b) which show that these constructions can be extended to any simply connected nilpotent Lie group and can be related to the program of Weyl quantization for coadjoint orbits (see e.g., Wi89] and [Ca07]) and to the Weyl calculus for irreducible unitary representations discussed in Section 3 ,

A magnetic potential on a Lie group $G$ is simply a 1 -form $A \in \Omega^{1}(G)$, and the corresponding magnetic field is $B=d A \in \Omega^{2}(G)$. The purpose of a magnetic pseudo-differential calculus on $G$ is to facilitate the investigation on first-order linear differential operators of the form $-\mathrm{i} P_{0}+A(Q) P_{0}$, where $P_{0}$ is a right invariant vector field on $G$ and $A(Q) P_{0}$ stands for the operator defined by the multiplication by the function obtained by applying the (non-invariant) 1-form $A$ to the vector field $P_{0}$ at every point in $G$. In the special case of the abelian group $G=\left(\mathbb{R}^{n},+\right)$ we have $A=A_{1} \mathrm{~d} x_{1}+\cdots+A_{n} \mathrm{~d} x_{n} \in \Omega^{1}\left(\mathbb{R}^{n}\right)$ and the above operators are precisely the linear partial differential operators determined by vectors $P_{0}=\left(p_{1}, \ldots, p_{n}\right) \in \mathbb{R}^{n}$,

$$
\mathrm{i}\left(p_{1} \frac{\partial}{\partial x_{1}}+\cdots+p_{n} \frac{\partial}{\partial x_{n}}\right)+\left(p_{1} A_{1}(Q)+\cdots+p_{n} A_{n}(Q)\right)=\sum_{j=1}^{n} p_{j}\left(\mathrm{i} \frac{\partial}{\partial x_{j}}+A_{j}(Q)\right)
$$

where we denote by $A_{1}(Q), \ldots, A_{n}(Q)$ the operators of multiplication by the coefficients of the 1-form $A$. In the non-magnetic case (i.e., $d A=B=0$ ), we get precisely the operators involved in the classical Weyl calculus on $\mathbb{R}^{n}$ discussed in Section 2 . 


\subsection{Magnetic Weyl calculus.}

Setting 4.1. Let us summarize the framework for the present section.

(1) A connected, simply connected, nilpotent Lie group $G$ is identified to its Lie algebra $\mathfrak{g}$ by means of the exponential map. We denote by $*$ the Baker-Campbell-Hausdorff multiplication on $\mathfrak{g}$, so that $G=(\mathfrak{g}, *)$.

(2) The cotangent bundle $T^{*} G$ is a trivial bundle and we perform the identification

$$
T^{*} G \simeq \mathfrak{g} \times \mathfrak{g}^{*}
$$

by using the trivialization by left translations.

(3) $\mathscr{F}$ is an admissible function space on the Lie group $G$ (see Def. 2.8 in [BB09a]); in particular, $\mathscr{F}$ is invariant under translations to the left on $G$ and is endowed with a locally convex topology such that we have continuous inclusion mappings $\mathfrak{g}^{*} \hookrightarrow \mathscr{F} \hookrightarrow \mathscr{C}^{\infty}(G)$. For instance $\mathscr{F}$ can be the whole space $\mathscr{C}^{\infty}(G)$ or the space $\mathscr{C}_{\text {pol }}^{\infty}(G)$ of smooth functions with polynomial growth. See however $[\mathrm{BB} 09 \mathrm{~b}]$ for specific situations when $\operatorname{dim} \mathscr{F}<\infty$.

(4) The semidirect product $M=\mathscr{F} \rtimes_{\lambda} G$ is an infinite-dimensional Lie group in general, whose Lie algebra is $\mathfrak{m}=\mathscr{F} \rtimes_{\dot{\lambda}} \mathfrak{g}$. We refer to [Ne06 for basic facts on infinite-dimensional Lie groups.

(5) We endow $\mathfrak{g}$ and its dual space $\mathfrak{g}^{*}$ with Lebesgue measures suitably normalized such that the Fourier transform $L^{2}(\mathfrak{g}) \rightarrow L^{2}\left(\mathfrak{g}^{*}\right)$ is a unitary operator, and we denote $\mathscr{H}=L^{2}(\mathfrak{g})$.

(6) We define a unitary representation $\pi: M \rightarrow \mathscr{B}(\mathscr{H})$ by

$$
(\pi(\phi, X) f)(Y)=\mathrm{e}^{\mathrm{i} \phi(Y)} f((-X) * Y)
$$

for $(\phi, X) \in M, f \in \mathscr{H}$, and $Y \in \mathfrak{g}$.

(7) The magnetic potential $A \in \Omega^{1}(G)$ is a smooth differential 1-form whose coefficients belong to $\mathscr{F}$. That is, a smooth mapping $A: \mathfrak{g} \rightarrow \mathfrak{g}^{*}, X \mapsto A_{X}$, with polynomial growth such that for every $X \in \mathfrak{g}$ we have $\left\langle A_{\bullet},\left(R_{\bullet}\right)_{0}^{\prime} X\right\rangle \in \mathscr{F}$. The corresponding magnetic field is the 2 -form $B=d A \in \Omega^{2}(G)$. Hence $B$ is a smooth mapping $X \mapsto B_{X}$ from $\mathfrak{g}$ into the space of all skewsymmetric bilinear functionals on $\mathfrak{g}$ such that

$$
\left(\forall X, X_{1}, X_{2} \in \mathfrak{g}\right) \quad B_{X}\left(X_{1}, X_{2}\right)=\left\langle A_{X}^{\prime}\left(X_{1}\right), X_{2}\right\rangle-\left\langle A_{X}^{\prime}\left(X_{2}\right), X_{1}\right\rangle .
$$

(8) We also need the mappings

$$
\theta_{0}: \mathfrak{g} \times \mathfrak{g}^{*} \rightarrow \mathscr{F}, \quad \theta_{0}(X, \xi)=\xi+\left\langle A_{\bullet},(R \bullet)_{0}^{\prime} X\right\rangle
$$

and

$$
\theta: \mathfrak{g} \times \mathfrak{g}^{*} \rightarrow \mathfrak{m}, \quad(X, \xi) \mapsto\left(\theta_{0}(X, \xi), X\right) .
$$

Here $R_{Y}: \mathfrak{g} \rightarrow \mathfrak{g}, Z \mapsto Z * Y$, is the translation to the right defined by any $Y \in \mathfrak{g}$.

We refer to BB09a] for more details on these notions.

Remark 4.2. Let $\Xi:=\mathfrak{g} \times \mathfrak{g}^{*}$ and denote the duality between $\mathfrak{g}$ and $\mathfrak{g}^{*}$ also by

$$
\mathfrak{g}^{*} \times \mathfrak{g} \rightarrow \mathbb{R}, \quad(\xi, X) \mapsto\langle\xi, X\rangle .
$$

The mapping $\langle\cdot, \cdot\rangle: \Xi \times \Xi \rightarrow \mathbb{R}, \quad\left\langle\left(X_{1}, \xi_{1}\right),\left(X_{2}, \xi_{2}\right)\right\rangle=\left\langle\xi_{1}, X_{2}\right\rangle-\left\langle\xi_{2}, X_{1}\right\rangle$ defines a symplectic structure on $\Xi$. The corresponding Fourier transform is given by

$$
\left(F_{\Xi} a\right)(X, \xi)=\hat{a}(X, \xi)=\int_{\Xi} \mathrm{e}^{-\mathrm{i}\langle(X, \xi),(Y, \eta)\rangle} a(Y, \eta) \mathrm{d}(Y, \eta), \quad a \in L^{1}(\Xi) .
$$

This transform extends to an invertible operator $\mathscr{S}^{\prime}(\Xi) \rightarrow \mathscr{S}^{\prime}(\Xi), F_{\Xi}^{-1}=F_{\Xi}$ and we denote $\check{a}=$ $F_{\Xi}^{-1} a$. 
In the present framework we can make the following definition similar to Definition 3.6. To emphasize the close relationship between these two constructions, let us mention that the representation $\pi$ introduced in Setting 4.1(6) is naturally associated with a certain finite-dimensional coadjoint orbit $\mathscr{O}$ of the infinitedimensional Lie group $M=\mathscr{F} \rtimes_{\lambda} G$ and there exists a canonical symplectomorphism $\mathscr{O} \simeq T^{*} G$. (See Prop. 2.9 and subsect 2.4 in BB09a.)

Definition 4.3. For every $a \in \mathscr{S}\left(\mathfrak{g} \times \mathfrak{g}^{*}\right)$ there exists a linear operator $\operatorname{Op}^{A}(a)$ in $L^{2}(\mathfrak{g})$ defined by

$$
\mathrm{Op}^{A}(a) f=\int_{\Xi} \check{a}(X, \xi) \pi\left(\exp _{M} \theta(X, \xi)\right) f \mathrm{~d}(X, \xi)
$$

We will call $\mathrm{Op}^{A}(a)$ a magnetic pseudo-differential operator with respect to the magnetic potential $A$. The function $a$ is the magnetic Weyl symbol of the pseudo-differential operator $\mathrm{Op}^{A}(a)$, and the Weyl calculus with respect to the magnetic potential $A$ is the mapping $\mathrm{Op}^{A}$ which takes a function $a \in \mathscr{S}\left(\mathfrak{g} \times \mathfrak{g}^{*}\right)$ into the corresponding pseudo-differential operator. By using a duality reasoning (see [BB09b]), one can extend this definition to every tempered distribution $a \in \mathscr{S}^{\prime}\left(\mathfrak{g} \times \mathfrak{g}^{*}\right)$ in order to get a continuous linear pseudo-differential operator $\operatorname{Op}^{A}(a): \mathscr{S}(\mathfrak{g}) \rightarrow \mathscr{S}^{\prime}(\mathfrak{g})$.

Remark 4.4. The role of $\mathfrak{g}_{e}$ of Definition 3.6 is played here by the magnetic predual

$$
\mathscr{O}_{*}^{A}:=\left\{\left(\bar{\theta}_{0}^{A}(\xi, X), X\right) \mid X \in \mathfrak{g}, \xi \in \mathfrak{g}^{*}\right\} \subseteq \mathscr{F} \rtimes_{\dot{\lambda}} \mathfrak{g}=\mathfrak{m}
$$

for the orbit $\mathscr{O}$. The set $\mathscr{O}_{*}^{A}$ is just a "copy" of $\mathscr{O}$ contained in the Lie algebra $\mathfrak{m}$ of the infinite-dimensional Lie group $M$. In the general case, if two magnetic potentials give rise to the same magnetic field, then the corresponding copies of $\mathscr{O}$ in the Lie algebra $\mathfrak{m}$ are moved to each other by the adjoint action of the Lie group $M$ (see Rem. 3.5 in [BB09a ). This leads to the gauge covariance (Theorem 4.5)(2) below) of the pseudo-differential calculus which can also be described by the formula

$$
\mathrm{Op}^{A}(a) f=\int_{\mathscr{O}_{*}} \check{a}(v) \pi\left(\exp _{M} v\right) f \mathrm{~d} v
$$

obtained from (4.1) after the change of variables $v=\theta(x, \xi)$.

Theorem 4.5. The Weyl calculus $\mathrm{Op}^{A}$ has the following properties:

(1) For $P_{0} \in \mathfrak{g}$ let us denote by $A(Q) P_{0}$ the multiplication operator defined by the function $Y \mapsto$ $\left\langle A_{Y},\left(R_{Y}\right)_{0}^{\prime} P_{0}\right\rangle$. Then the usual functional calculus for the self-adjoint operator $-\mathrm{i} \dot{\lambda}\left(P_{0}\right)+A(Q) P_{0}$ in $L^{2}(\mathfrak{g})$ can be recovered from $\mathrm{Op}^{A}$.

(2) Gauge covariance with respect to the magnetic potential $A$ : If $A_{1} \in \Omega^{1}(\mathfrak{g})$ is another magnetic potential with $d A=d A_{1} \in \Omega^{2}(\mathfrak{g})$ and the function $Y \mapsto\left\langle A_{Y},\left(R_{Y}\right)_{0}^{\prime} X\right\rangle$ belongs to $\mathscr{F}$ for every $X \in \mathfrak{g}$, then there exists $\psi \in \mathscr{F}$ such that unitary operator $U: L^{2}(\mathfrak{g}) \rightarrow L^{2}(\mathfrak{g})$ defined by the multiplication by the function $\mathrm{e}^{\mathrm{i} \psi}$ satisfies the condition $\mathrm{UOp}^{A}(a) U^{-1}=\mathrm{Op}^{A_{1}}(a)$ for every symbol $a \in \mathscr{S}\left(\mathfrak{g} \times \mathfrak{g}^{*}\right)$.

(3) If $\mathscr{C}_{\text {pol }}^{\infty}(\mathfrak{g}) \subseteq \mathscr{F}$ and the function $Y \mapsto\left\langle A_{Y},\left(R_{Y}\right)_{0}^{\prime} X\right\rangle$ belongs to $\mathscr{C}_{\text {pol }}^{\infty}(\mathfrak{g})$ for every vector $X \in \mathfrak{g}$, then for every symbol $a \in \mathscr{S}\left(\mathfrak{g} \times \mathfrak{g}^{*}\right)$ the magnetic pseudo-differential operator $\mathrm{Op}^{A}(a)$ is bounded linear on $L^{2}(\mathfrak{g})$ and is defined by an integral kernel $K_{a} \in \mathscr{S}(\mathfrak{g} \times \mathfrak{g})$ given by formula

$$
K_{a}(X, Y)=\alpha_{A}(X, Y) \int_{\mathfrak{g}^{*}} \mathrm{e}^{\mathrm{i}\langle\xi, X *(-Y)\rangle} a\left(\int_{0}^{1}(s(Y *(-X))) * X \mathrm{~d} s, \xi\right) \mathrm{d} \xi
$$


were we have used the notation

$$
\alpha_{A}(X, Y)=\exp \left(\mathrm{i} \int_{0}^{1}\left\langle A((s(Y *(-X))) * X),\left(R_{(s(Y *(-X))) * X}\right)_{0}^{\prime}(X *(-Y))\right\rangle \mathrm{d} s\right)
$$

for every $X, Y \in \mathfrak{g}$.

(4) Under the hypothesis of the above Assertion [3, the correspondence $a \mapsto K_{a}$ is an isomorphism of Fréchet spaces $\mathscr{S}\left(\mathfrak{g} \times \mathfrak{g}^{*}\right) \rightarrow \mathscr{S}(\mathfrak{g} \times \mathfrak{g})$ and extends to a unitary operator $L^{2}\left(\mathfrak{g} \times \mathfrak{g}^{*}\right) \rightarrow L^{2}(\mathfrak{g} \times \mathfrak{g})$.

(5) If the distribution $a \in \mathscr{S}^{\prime}\left(\mathfrak{g} \times \mathfrak{g}^{*}\right)$ takes real values on the real valued functions, then the pseudodifferential operator $\mathrm{Op}^{A}(a)$ is symmetric, in the sense that its distribution kernel $K_{a} \in \mathscr{S}^{\prime}(\mathfrak{g} \times \mathfrak{g})$ has the following symmetry property:

$$
(\forall f, \phi \in \mathscr{S}(\mathfrak{g})) \quad\left\langle K_{a}, f \otimes \bar{\phi}\right\rangle=\overline{\left\langle K_{a}, \phi \otimes \bar{f}\right\rangle} .
$$

Proof. See $\mathrm{BB} 09 \mathrm{a}]$ and $\mathrm{BB} 09 \mathrm{~b}]$.

\subsection{Magnetic Moyal product.}

Definition 4.6. Let us assume that $\mathscr{C}_{\text {pol }}^{\infty}(\mathfrak{g}) \subseteq \mathscr{F}$ and $A \in \Omega^{1}(\mathfrak{g})$ has the property that the function $Y \mapsto\left\langle A_{Y},\left(R_{Y}\right)_{0}^{\prime} X\right\rangle$ belongs to $\mathscr{C}_{\text {pol }}^{\infty}(\mathfrak{g})$ for every $X \in \mathfrak{g}$. It follows by Theorem 4.5(3) that for every $a_{1}, a_{2} \in \mathscr{S}\left(\mathfrak{g} \times \mathfrak{g}^{*}\right)$ there exists a unique function $a_{1} \#^{A} a_{2} \in \mathscr{S}\left(\mathfrak{g} \times \mathfrak{g}^{*}\right)$ such that $\mathrm{Op}^{A}\left(a_{1}\right) \mathrm{Op}^{A}\left(a_{2}\right)=$ $\mathrm{Op}^{A}\left(a_{1} \#^{A} a_{2}\right)$ and the magnetic Moyal product

$$
\mathscr{S}\left(\mathfrak{g} \times \mathfrak{g}^{*}\right) \times \mathscr{S}\left(\mathfrak{g} \times \mathfrak{g}^{*}\right) \rightarrow \mathscr{S}\left(\mathfrak{g} \times \mathfrak{g}^{*}\right), \quad\left(a_{1}, a_{2}\right) \mapsto a_{1} \#^{A} a_{2}
$$

is a bilinear continuous mapping.

Theorem 4.7. Let $\mathfrak{g}$ be a two-step nilpotent Lie algebra, that is, $[\mathfrak{g},[\mathfrak{g}, \mathfrak{g}]]=\{0\}$. If $\mathscr{C}_{\text {pol }}^{\infty}(\mathfrak{g}) \subseteq \mathscr{F}$ and $A \in \Omega^{1}(\mathfrak{g})$ is a magnetic potential with $\left\langle A(\cdot), X+\frac{1}{2}[X, \cdot]\right\rangle \in \mathscr{C}_{\text {pol }}^{\infty}(\mathfrak{g})$ for every $X \in \mathfrak{g}$, then the following assertions hold:

(1) For every $a \in \mathscr{S}\left(\mathfrak{g} \times \mathfrak{g}^{*}\right)$ the integral kernel of the bounded linear operator $\mathrm{Op}^{A}(a): L^{2}(\mathfrak{g}) \rightarrow L^{2}(\mathfrak{g})$ is given by the formula

$$
K_{a}(X, Y)=\alpha_{A}(X, Y) \int_{\mathfrak{g}^{*}} \mathrm{e}^{\mathrm{i}\langle\xi, X *(-Y)\rangle} a\left(\frac{1}{2}(X+Y), \xi\right) \mathrm{d} \xi
$$

where for arbitrary $X, Y \in \mathfrak{g}$ we have denoted

$$
\alpha_{A}(X, Y)=\exp \left(-\mathrm{i} \int_{0}^{1}\langle A(s Y+(1-s) X), Y-X\rangle \mathrm{d} s\right) .
$$

(2) Set

$$
\beta_{A}: \mathfrak{g} \times \mathfrak{g} \times \mathfrak{g} \rightarrow \mathbb{C}, \quad \beta_{A}(X, Y, Z)=\alpha_{A}^{-1}(X, Y) \alpha_{A}(Y, Z) \alpha_{A}(Z, X) .
$$

If $a, b \in \mathscr{S}\left(\mathfrak{g} \times \mathfrak{g}^{*}\right)$ then for every $(X, \xi) \in \mathfrak{g} \times \mathfrak{g}^{*}$ we have

$$
\begin{array}{rl}
\left(a \#^{A} b\right)(X, \xi)=\iiint \int_{\mathfrak{g} \times \mathfrak{g} \times \mathfrak{g}^{*} \times \mathfrak{g}^{*}} & a(Z, \zeta) b(T, \tau) \mathrm{e}^{2 \mathrm{i}\langle(Z-X, \zeta-\xi),(T-X, \tau-\xi)\rangle} \\
& \times \mathrm{e}^{-\mathrm{i}(\langle\xi+\zeta,[X, Z]\rangle+\langle\zeta+\tau,[Z, T]\rangle+\langle\tau+\xi,[T, X]\rangle)} \\
& \times \beta_{A}(Z-T+X, T-Z+X, Z+T-X) \mathrm{d} Z \mathrm{~d} T \mathrm{~d} \zeta \mathrm{d} \tau .
\end{array}
$$

Proof. See $[\mathrm{BB} 09 \mathrm{a}]$. 
Acknowledgments. Partial financial support from the grant PNII - Programme "Idei" (code 1194) is acknowledged.

\section{REFERENCES}

[An69] R.F.V. Anderson, The Weyl functional calculus. J. Functional Analysis 4(1969), 240-267.

[An72] R.F.V. Anderson, The multiplicative Weyl functional calculus. J. Functional Analysis 9(1972), 423-440.

[BB09a] I. Beltiţă, D. Beltiţă, Magnetic pseudo-differential Weyl calculus on nilpotent Lie groups. Ann. Global Anal. Geom. 36 (2009) no. 3, 293-322.

[BB09b] I. Beltiţă, D. Beltiţă, Uncertainty principles for magnetic structures on certain coadjoint orbits. J. Geom. Phys. (to appear).

[BB09c] I. Beltiţă, D. Beltiţă, Modulation spaces of symbols for representations of nilpotent Lie groups. Preprint, arXiv:0908.3917 2 [math.AP].

[Ca07] B. CAhen, Weyl quantization for semidirect products. Differential Geom. Appl. 25 (2007), no. 2, 177-190.

[FG92] H.G. Feichtinger, K. Gröchenig, Gabor wavelets and the Heisenberg group: Gabor expansions and short time Fourier transform from the group theoretical point of view. In: Wavelets, Wavelet Anal. Appl., 2, Academic Press, Boston, MA, 1992, pp. 359-397.

[G107] P. GŁowACKI, The Melin calculus for general homogeneous groups. Ark. Mat. 45 (2007), no. 1, 31-48.

[Fo89] G.B. Folland, Harmonic Analysis in Phase Space. Annals of Mathematics Studies, 122. Princeton University Press, Princeton, NJ, 1989.

[dG06] M. DE Gosson, Symplectic Geometry and Quantum Mechanics. Operator Theory: Advances and Applications, 166. Advances in Partial Differential Equations (Basel). Birkhäuser Verlag, Basel, 2006.

[Gr01] K. Gröchenig, Foundations of Time-Frequency Analysis. Applied and Numerical Harmonic Analysis. Birkhäuser Boston, Inc., Boston, MA, 2001.

[GH99] K. Gröchenig, C. Heil, Modulation spaces and pseudodifferential operators. Integral Equations Operator Theory 34 (1999), no. 4, 439-457.

[HN85] B. Helffer, J. Nourrigat, Hypoellipticité Maximale pour des Opérateurs Polynômes de Champs de Vecteurs. Progress in Mathematics, 58. Birkhäuser Boston, Inc., Boston, MA, 1985.

[Hor79] L. Hörmander, The Weyl calculus of pseudodifferential operators. Comm. Pure Appl. Math. 32 (1979), no. 3, 360-444.

[Hor07] L. Hörmander, The Analysis of Linear Partial Differential Operators. III. Pseudo-differential operators. Reprint of the 1994 edition. Classics in Mathematics. Springer, Berlin, 2007.

[How80] R. Howe, On the role of the Heisenberg group in harmonic analysis. Bull. Amer. Math. Soc. (N.S.) 3 (1980), no. $2,821-843$.

[How84] R.E. Howe, A symbolic calculus for nilpotent groups. In: Operator Algebras and Group Representations (Neptun, 1980), vol. I, Monogr. Stud. Math., 17, Pitman, Boston, MA, 1984, pp. 254-277.

[HRW84] R. Howe, G. RAtcliff, N. Wildberger, Symbol mappings for certain nilpotent groups. In: Lie group representations III (College Park, Md., 1982/1983), Lecture Notes in Math., 1077, Springer, Berlin, 1984,pp. 288-320.

[IMP07] V. Iftimie, M. Măntoiu, R. Purice, Magnetic pseudodifferential operators. Publ. Res. Inst. Math. Sci. 43 (2007), no. 3, 585-623.

[Kir62] A.A. Kirillov, Unitary representations of nilpotent Lie groups. (Russian) Uspehi Mat. Nauk 17 (1962), no. 4 (106), 57-110.

[KT03] A.E. Krasowska, S.T. Ali, Wigner functions for a class of semi-direct product groups. J. Phys. A 36 (2003), no. $11,2801-2820$.

[Ma07] J.-M. MAILlARD, Explicit star products on orbits of nilpotent Lie groups with square integrable representations. $J$. Math. Phys. 48 (2007), no. 7, 073504.

[Ma91] D. Manchon, Calcul symbolique sur les groupes de Lie nilpotents et applications. J. Funct. Anal. 102 (1991), no. $1,206-251$.

[Ma95] D. Manchon, Opérateurs pseudodifférentiels et représentations unitaires des groupes de Lie. Bull. Soc. Math. France 123 (1995), no. 1, 117-138.

[MP04] M. Măntoiu, R. Purice, The magnetic Weyl calculus. J. Math. Phys. 45 (2004), no. 4, 1394-1417.

[Me83] A. Melin, Parametrix constructions for right invariant differential operators on nilpotent groups. Ann. Global Anal. Geom. 1 (1983), no. 1, 79-130.

[Mi82] K.G. Miller, Invariant pseudodifferential operators on two-step nilpotent Lie groups. Michigan Math. J. 29 (1982), no. $3,315-328$.

[Mi86] K.G. MiLler, Invariant pseudodifferential operators on two-step nilpotent Lie groups. II. Michigan Math. J. 33 (1986), no. 3, 395-401.

[Ne06] K.-H. NeEB, Towards a Lie theory of locally convex groups. Japanese J. Math. 1 (2006), no. 2, 291-468. 
[Pe84] N.V. Pedersen, On the infinitesimal kernel of irreducible representations of nilpotent Lie groups. Bull. Soc. Math. France 112 (1984), no. 4, 423-467.

[Pe88] N.V. Pedersen, On the symplectic structure of coadjoint orbits of (solvable) Lie groups and applications. I. Math. Ann. 281 (1988), no. 4, 633-669.

[Pe89] N.V. Pedersen, Geometric quantization and the universal enveloping algebra of a nilpotent Lie group. Trans. Amer. Math. Soc. 315 (1989), no. 2, 511-563.

[Pe94] N.V. Pedersen, Matrix coefficients and a Weyl correspondence for nilpotent Lie groups. Invent. Math. 118 (1994), no. $1,1-36$.

[We28] H. WeYL, Gruppentheorie und Quantenmechanik. Verlag S. Hirzel, Leipzig, 1928.

[Wi89] N.J. Wildberger, Convexity and unitary representations of nilpotent Lie groups. Invent. Math. 98 (1989), no. 2, $281-292$.

Institute of Mathematics "Simion Stoilow" of the Romanian Academy, P.O. Box 1-764, Bucharest, Romania

E-mail address: Ingrid.Beltita@imar.ro

E-mail address: Daniel.Beltita@imar.ro 